\title{
Development of a Measurement System of a Body Conduction Sound using Wireless LAN
}

\author{
Fumiaki Ehara ${ }^{\mathrm{a},{ }^{*}}$, Ryouhei Tanaka ${ }^{\mathrm{a}}$, Kanya Tanaka ${ }^{\mathrm{b}}$

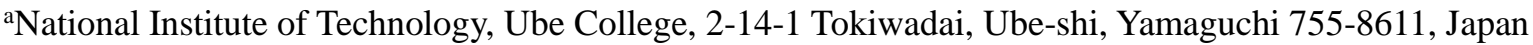 \\ bYamaguchi University, 2-16-1 Tokiwadai, Ube-shi, Yamaguchi 755-8611, Japan \\ *Corresponding Author: ehara@ube-k.ac.jp
}

\begin{abstract}
Solitary death of elderly people is recognized as social problem in Japan. There is a real need for a new device to monitor the health condition of elderly people. In this study, we made a health monitoring system by measuring a body conduction sound. The system consists of a measurement device and a server. The device consists of a body conduction sound sensor, a microcontroller and a Wi-Fi module. The server indicates the measurement result as a graph and derives the pulse rate from the autocorrelation result of the measurement data. The measurement data are sent to the separated server using Wireless LAN. The efficacy of the system is confirmed by the measurement of a body conduction sound.
\end{abstract}

Keywords: body conduction sound, Wi-Fi, mbed.

\section{Introduction}

Solitary death of elderly people is recognized as social problem in Japan. It is difficult to substantiate a system which monitor them using camera because of lack of privacy. There is a real need for a new monitoring system that conscious of privacy. Body conduction sounds are used to find an abnormality in body in medicine field. A sound that produced and propagated inside of the body is measured by a body conduction sound sensor that is put on the body surface. The doctor listens to the sound through a stethoscope, and uses it to discovery of the illness. Some researchers reported a system to find unusual sounds of the heart $^{(1,2)}$, and an analysis method was reported that finds an abnormal cardiac sound measured by the wireless electronic stethoscope $^{(3)}$. A system using NAM (non-audible murmur) microphones was also reported ${ }^{(4,5)}$. NAM microphone was developed to detect a very weak speech sound. NAM microphone can be produced more cheaply than the electronic stethoscope, and has an advantage in commercialization. In recent years, development of microcomputers makes it possible easier and more cheaply to structure a measurement system than before. It is expected that a system which examines the health condition at a distant place, which diagnoses an internal disease are developed by combining the body conduction sound sensor with a microcomputer, and the system would be small and not get in the way of daily life.

In this paper, we made a health monitoring system by measuring a body conduction sound. The system consists of a measurement device and a server. The device consists of a body conduction sound sensor, a microcontroller and a WiFi module. The body conduction sound sensor are made using electret condenser microphone (ECM). The measurement data are sent to the separated server using Wireless LAN. The server program are made in Visual C\#. The server filters out the burst noise from the measurement data using median filter, indicates the measurement result as a graph and derives the pulse rate from the autocorrelation result of the measurement data. The efficacy of the system is confirmed by the measurement of a body conduction sound.

\section{Measurement System}

\subsection{Body conduction sound sensor}

The body conduction sound sensor is made by use of an improved small ECM (Primo Co., EM189T). The microphone whose case is drilled a hole in and diaphragm is exposed is used. Figure 1 shows the container of the sensor that is made using acrylic as a material. The microphone is fixed in the center of the container, filled up with 


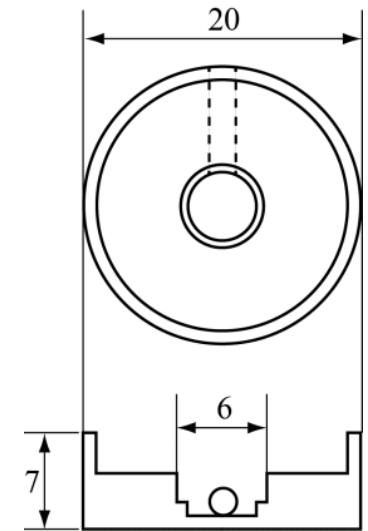

Fig. 1. Structure of the container of the sensor.
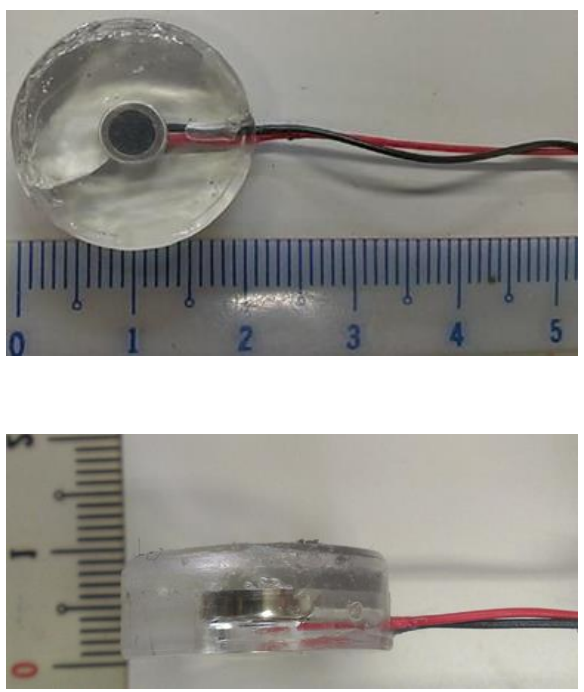

Fig. 2. The body conduction sound sensor.

polyurethane resin. Then it can detect sounds inside of the body. Figure 2 is a picture of the sensor.

\section{2 mbed and Wi-Fi}

In this study, a mbed LPC1768 is used to control the measurement system. The mbed is an open-source electronics prototyping platform which consists of a 32 bit microcontroller on the board, a programming language and its development environment. Table 1 shows specifications of the mbed LPC1768. It is a microcontroller board based on the ARM Cortex-M3 microcontroller. It has 26 digital inputoutput pins and 6 analog inputs. It connects to a computer with a USB cable and we can write a program using the connection. Its development environment is provided on the Web. It doesn't require a special application to develop the mbed program, we can do well with a web-connected computer.

It has more coping skills than the other 8 bit microcontrollers. And the amount of memory installed is
Table 1. Specifications of mbed LPC1768

\begin{tabular}{|c|c|}
\hline Core & ARM Cortex-M3 \\
\hline Frequency & $96 \mathrm{MHz}$ \\
\hline Flash Memory & $512 \mathrm{~KB}$ \\
\hline RAM & $32 \mathrm{~KB}$ \\
\hline Supply Voltage & $4.5-9.0 \mathrm{~V}$ \\
\hline Digital I/O Pins & 26 \\
\hline Analog Input Pins & 6 \\
\hline PWM Output Pins & 8 \\
\hline Analog Output Pins & 1 \\
\hline
\end{tabular}

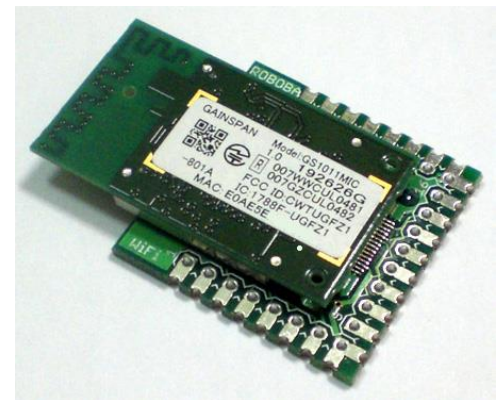

Fig. 3. GainSpan GS1011MIC.

Table 2. Specifications of GS1011MIC

\begin{tabular}{|c|c|}
\hline Radio Protocol & IEEE $802.11 \mathrm{~b} / \mathrm{g} / \mathrm{n}$ \\
\hline RF Output Power & $9 \mathrm{dBm}$ \\
\hline Supported Data Rates & $11,5.5,2,1 \mathrm{Mbps}(802.11 \mathrm{~b})$ \\
\hline Security Protocols & WEP, WPA, WPA2, WPS \\
\hline Network Protocol & $\begin{array}{c}\text { ARP, UDP, TCP, DHCP, } \\
\text { DNS, NTP, HTP/HTTPS } \\
(\text { IPv4) }\end{array}$ \\
\hline $\begin{array}{c}\text { Outline Dimensions } \\
(\mathrm{mm})\end{array}$ & $35 \times 20 \times 3.4$ \\
\hline
\end{tabular}

larger than the other 8 bit microcontrollers, it is good to deal with sound data. It has the flexibility of lots of peripheral interfaces, such as Ethernet and USB.

To observe from a distance, wireless radio communication is used between the mbed and a computer. In this study, wireless LAN (Wi-Fi) is used as a means of communication. Wi-Fi is more popular than XBee and Bluetooth which can be used as a radio communication device. Figure 3 shows the Wi-Fi module (Gainspan, GS1011MIC) and Table 2 shows the specifications. The module is low power consumption and suitable for our system. 


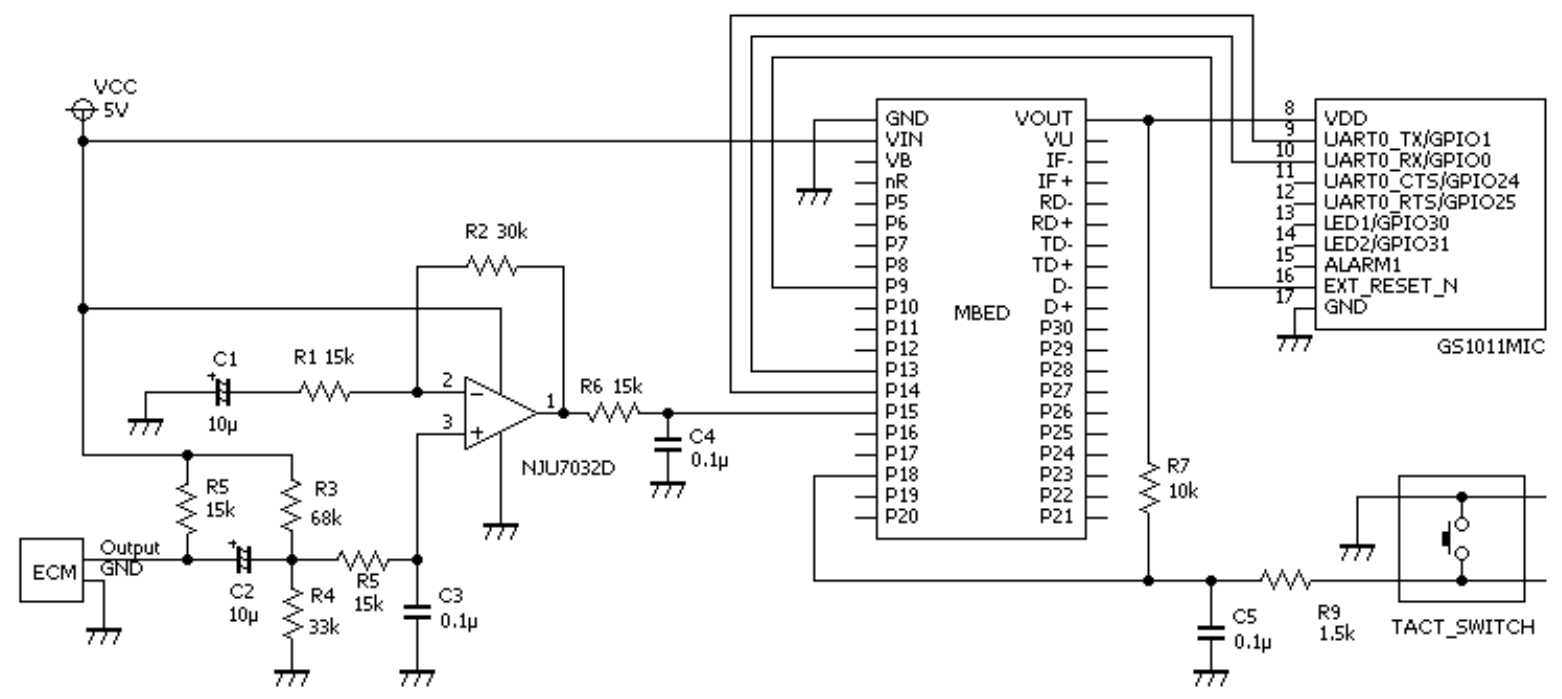

Fig. 4. Circuit diagram of the measurement system.

\section{3 measurement device}

Figure 4 shows the circuit diagram of the measurement system. Figure 5 shows the flowchart of the measurement program. A body conduction sound is measured at the flick of a switch using the sensor. The measured data are amplified by a non-inverting amplifier circuit. The data are sent to a computer by wireless LAN using GS1011MIC. Figure 6 shows the measurement device we made.

\subsection{Server}

The server program are made in Visual C\#. It is able to get the measurement result in wireless radio communication and indicates them as a graph. Figure 7 shows the user interface of the server. It derives the pulse rate from the autocorrelation result of the measurement data, and the result also is indicated as a graph. The server filters out the burst noise from the measurement data using median filter.

Pulse rates are obtained to calculate the autocorrelation of the measured data. Autocorrelation shows repeated patterns of the signal, and shows the cycle length of a pulse rate. Equation 1 is the autocorrelation.

$$
\begin{array}{r}
R(j)=\frac{1}{N} \sum_{i=1}^{N} v(i) \cdot v(i+j) \\
(j=0,1,2, \cdots) .
\end{array}
$$

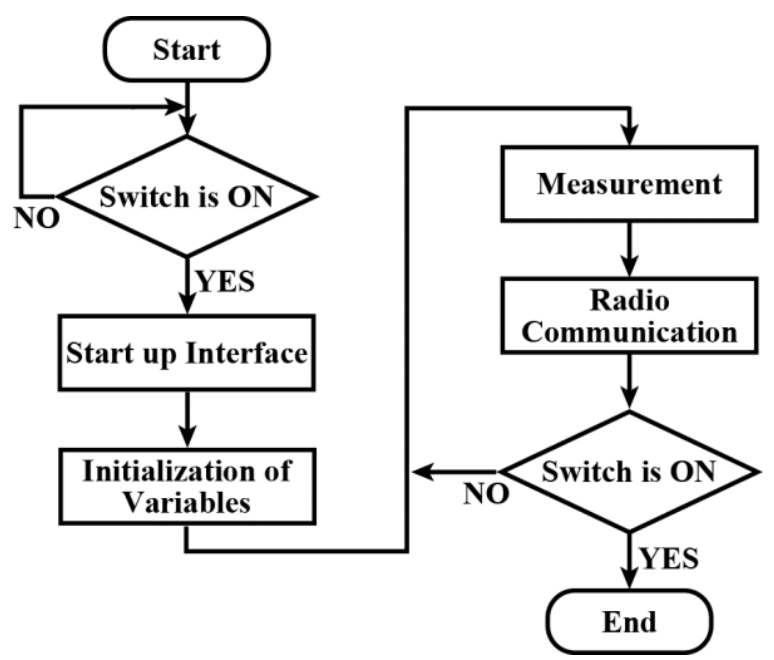

Fig. 5. Flowchart of the measurement program.

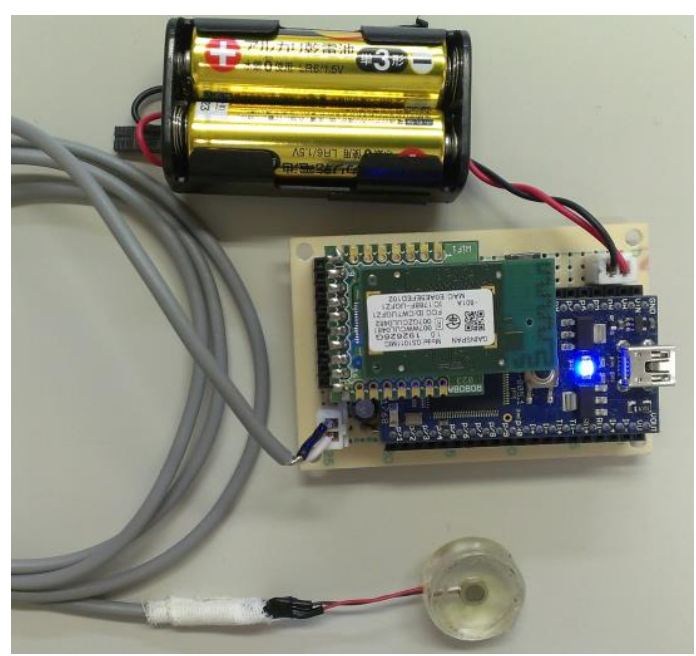

Fig. 6. Measurement device. 


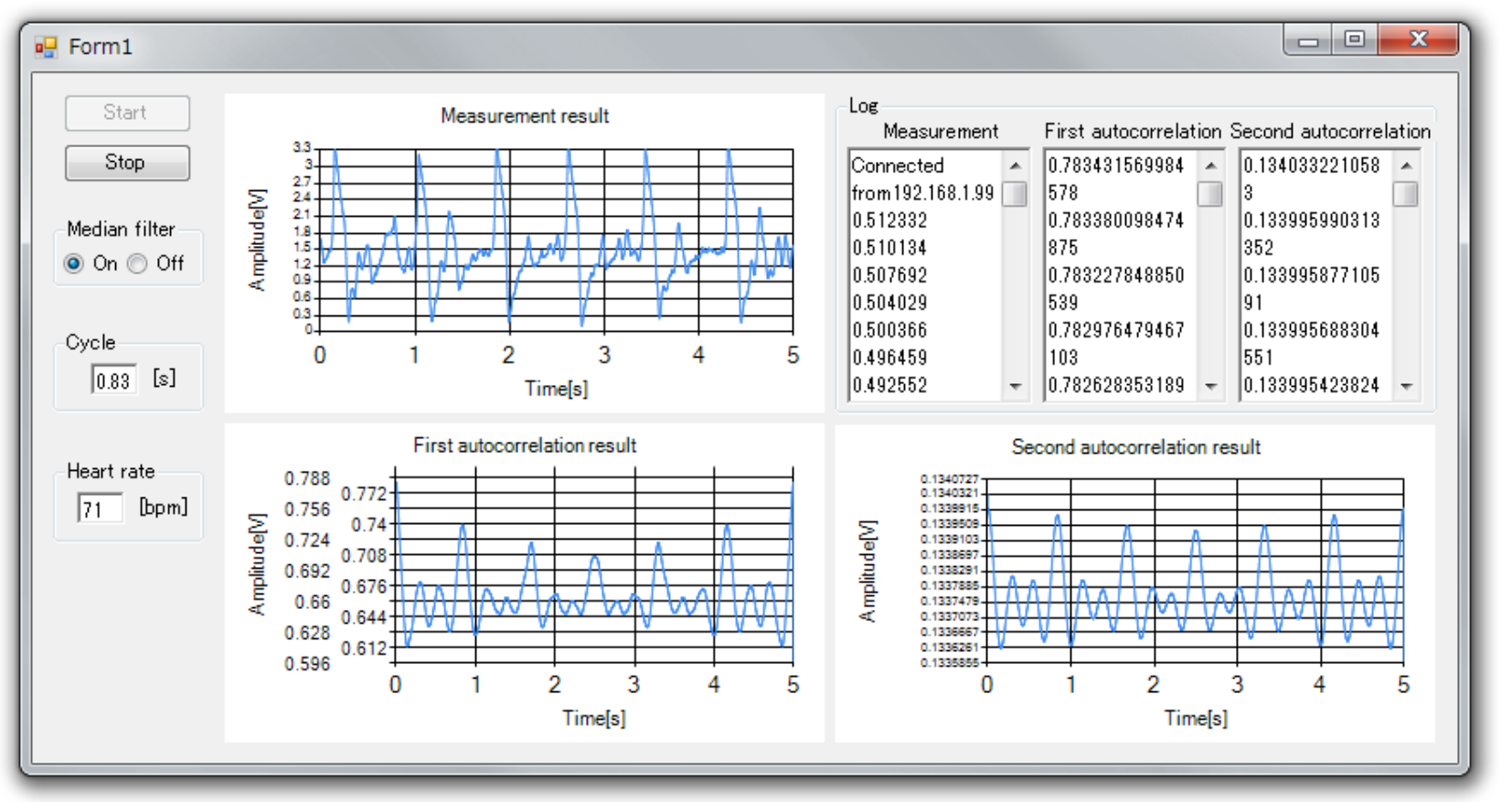

Fig. 7. User Interface of the server.

\section{Experiment}

To verify the effectiveness of this measurement system, we measured a body conduction sound using this system. An examinee (Male, 20s) was healthy and physically unimpaired, kept in a seated position at rest. We measured an arterial sound at the wrist. Sampling frequency is $1,000 \mathrm{~Hz}$, measurement time is 5 seconds (Fig.7).

In this experiment, the system is well-behaved. We compared two data, one is saved in mbed's memory after the measurement the other is sent by wireless LAN. They matched perfectly. We established that the system can accurately send the measurement data by wireless LAN.

\section{Conclusions}

In this paper, we made a health monitoring system by measuring a body conduction sound. The system consists of a measurement device and a server. The device consists of a body conduction sound sensor, a microcontroller and a WiFi module. The server indicates the measurement result as a graph and derives the pulse rate from the autocorrelation result of the measurement data.

To verify the effectiveness of this measurement system, we measured a body conduction sound using this system. We compared two data, one is saved in mbed's memory after the measurement the other is sent by wireless LAN. They matched perfectly. We established that the system can accurately send the measurement data by wireless LAN.

\section{References}

(1) J.R. Bulgrin, B.J. Rubal, C.R. Thompson and J.M. Moody : "Comparison of Short-Time Fourier, Wavelet and Time-Domain Analysis of Intracardiac Sounds", Biomed Sci. Instrum. Vol.29, pp.465-472, 1993

(2) C.H. Wu, C.W. Lo and J.F. Wang : "Computer-Aided Analysis and Classification of Heart Sounds Based on Neural Networks and Time Analysis", Proc. IEEE Int. Conf. Acoust. Speech Signal Process, pp.355-3458, 1995

(3) Z. Jiang and S. Choi : "Development of Wireless Electronic Stethoscope System and Abnormal Cradiac Sound Analysis Method (Sound Characteristic Waveform Analysis)", The Japan Society of Mechanical Engineers, pp.140-147, 2005

(4) T. Hirahara and S. Shimizu : "Sensing various types sound generated inside the body using an ECM-based compact body-conducted sound sensor", Proc. Autumn Meet. Acoust. Soc. Jpn., 2009

(5) S. Shimizu, M. Otani and T. Hirahara : "Frequency characteristics of several non-audible murmur (NAM) microphones", Acoust. Sci. \& Tech., pp.139-142, 2009 